\title{
A randomized clinical trial on arresting dentine caries in preschool children by topical fluorides - 18 month results
}

Short title: Arresting dentine caries in preschool children by fluorides

\section{Duangthip, C. H. Chu, E.C.M. Lo}

Faculty of Dentistry, The University of Hong Kong

\section{Email addresses:}

Duangporn DUANGTHIP: duangpornduangthip@gmail.com

Chun Hung CHU: chchu@hku.hk

Edward CM LO: edward-lo@hku.hk

Key words: Dental caries, Primary teeth, Preschool children, Fluorides, Clinical trial, Caries arrest

*Corresponding to : $\quad$ Edward C.M. Lo

3/F Prince Philip Dental Hospital

34 Hospital Road

Hong Kong

E-mail: edward-lo@hku.hk

Tel: +852-28590292

Fax: +852-28587874 


\title{
A randomized clinical trial on arresting dentine caries in preschool children by topical fluorides - 18 month results
}

\begin{abstract}
Objectives: This study aimed to compare the effectiveness of three topical fluoride application protocols in arresting dentine caries in primary teeth of preschool children.

Methods: Children aged 3-4 years who had at least one active dentine caries lesion were randomly allocated into three intervention groups: Group 1 - application of $30 \%$ silver diammine fluoride (SDF) solution every 12 months; Group 2 - three applications of 30\% SDF solution at weekly interval at baseline; and Group 3 - three applications of $5 \%$ sodium fluoride (NaF) varnish at weekly interval at baseline. A masked examiner carried out follow-up examinations every 6 months to assess whether the treated lesions had become arrested.
\end{abstract}

Results: A total of 304 children with 1670 tooth surfaces with dentine caries received treatment at baseline. After 18 months, 275 children (91\%) remained in the study. The caries arrest rates at tooth surface level were 40\%, 35\% and 27\% for Groups 1, 2 and 3, respectively ( $p<0.001)$. Result of the multi-level survival analysis showed that the two SDF application protocols could shorten the time to arrest of dentine caries compared with the NaF application protocol. Presence of plaque on lesion surface, tooth type and tooth surface all had significant effects on caries arrest rates.

Conclusions: Annual or three consecutive weekly applications of SDF solution is more effective in arresting dentine caries in primary teeth than three consecutive weekly applications of NaF varnish.

Clinical significance: In a water fluoridated area, application of SDF solution, either three weekly applications at baseline or annually, can arrest active dentine caries lesions in primary teeth faster than three weekly applications of NaF varnish at baseline. 


\section{Introduction}

Early childhood caries (ECC) is a global health problem that causes many children to suffer from pain and infection. ${ }^{1,2}$ Studies have shown that ECC is more prevalent among children in lower socio-economic groups and carious cavitated lesions in preschool children are mostly left untreated. ${ }^{3}$ Traditionally, a restorative approach which requires powered dental equipment and well trained dental personnel is adopted to treat the decayed teeth. The dental profession is currently faced with an enormous task of how to manage the heavy burden of untreated dental caries amongst the young child population. ${ }^{4}$

A number of clinical studies have shown that progression of dental caries in young children can be arrested by the use of silver diammine fluoride (SDF) solution. ${ }^{5-7}$ The commonly adopted frequency of application for caries arrest is once a year or every 6 months. ${ }^{5,8}$ The proportion of arrested caries increases with repeated application with SDF over time. To speed up the process of caries arrest, it would be logical to reduce the time interval between SDF applications. An intensive regimen of three applications of $10 \%$ SDF in three consecutive weeks was found to be effective in arresting enamel caries in permanent first molars. ${ }^{9}$ However, the sample size in that study is small, only 22 children aged 5-7 years were involved. The use of three consecutive weekly applications of $\mathrm{NaF}$ varnish was suggested for prevention of dental caries in young children. ${ }^{10}$ Using this approach with multiple applications in a short period, the treatment may be particularly beneficial for high caries risk populations as well as for migratory populations who are unable to follow a lengthy preventive program that requires regular visits.

So far, there is no published study which specifically investigates the efficacy of intensive applications of $\mathrm{NaF}$ varnish and SDF solution in arresting dentine caries in preschool children. Thus, the aim of this study was to compare the effectiveness of three topical fluoride application protocols in arresting dentine caries in primary teeth of high caries risk preschool children living in a fluoridated area. The null hypothesis to be tested was that the caries arrest rates of the lesions treated according to the three protocols were the same.

\section{Materials and Methods}

This study was conducted in 16 kindergartens in Hong Kong, where the drinking water has been fluoridated at a fluoride concentration of $0.5 \mathrm{ppm}$. Most of the toothpaste on sale in Hong Kong contain fluorides and those for children are fluoridated at 500 to 1000 ppm fluoride. 
Ethical approval was obtained from the Institutional Review Board of the University of Hong Kong and the trial was registered in the ClinicalTrials.gov registration system (no. NCT02426619). An invitation letter was sent to the parents explaining the purpose and procedures of this study. Written parental consent was received from the parents of all children. After screening, healthy children who had at least one tooth with untreated active dentine caries not involving pulp were invited to participate in the study. Clinical examination of the children was performed in the kindergarten by one dentist using disposable dental mirrors, lightemitting diode intra-oral illumination (MirrorLite) and ball-end probes (405/WHO CPI probe). The visible plaque index (VPI) and the dmfs index were used for recording the oral hygiene and dental caries status, respectively. At tooth surface level, status (inactive/active) of the dentine caries (ICDAS codes 5-6) ${ }^{11}$ and presence of plaque was assessed. Lesion activity was assessed by visual inspection and tactile detection. Light force was used and great care was taken to avoid damaging the tooth during probing. If the cavity wall or floor was easily penetrated by the ball-end probe using light force, it was diagnosed as active caries. Cavities with smooth and hard surfaces were classified as arrested caries. ${ }^{5,8,12}$ Tooth with a caries lesion extending into pulp or having signs that suggested it was non-vital, such as tooth discoloration, hypermobility and abscess, was not included in this study. Five surfaces (buccal, lingual, mesial, distal and occlusal) in each posterior tooth and four surfaces in each anterior teeth were assessed.

This clinical trial used a parallel group design. For ethical reasons, all active dental caries lesions were treated if possible and there was no negative control group. Participant children were put into two strata according to the number of carious tooth surfaces they had, one to four surfaces and $>4$ surfaces. They were then allocated by a stratified randomization method using a personal computer to one of the following three intervention groups:

Group 1: applications of a 30\% SDF solution (Cariestop, Biodinamica, Brazil) at baseline and every 12 months;

Group 2: 3 applications of a 30\% SDF solution at weekly interval at baseline; and Group 3: 3 applications of a 5\% NaF varnish (Duraphat, Colgate Palmolive, USA) at weekly interval at baseline.

A dentist who was not involved in the examination of the children applied the fluoride agents according to the child's group allocation. No effort was made to remove the carious tissues before fluoride application. Fluoride agent was placed in a plastic dappen dish. A 
disposable microbrush was used to apply the agent onto each carious lesion and rub for 10 seconds. After application, the child was asked not to drink or eat for at least 30 minutes. All examinations and treatments were carried out in the kindergartens without the presence of the children's parents.

At the 12-month follow-up, water was used as placebo in Groups 2 and 3. Follow-up examinations were conducted at 6,12 and18 months after baseline by the same masked examiner using the same equipment, procedure and diagnostic criteria. The examiner did not know the subjects' group allocation. In all examinations, a 10\% random sample of the children was re-examined for monitoring intra-examiner reproducibility.

Information about the children's demographic and socioeconomic background, and oral health related behaviors, and parent's satisfaction with the child's dental appearance was collected by a questionnaire completed by their parents at baseline. Sample size calculation was based on the results of previous studies which showed that around $70 \%$ of the active dental caries in preschool children became arrested after 24 months. ${ }^{5}$ An absolute difference of $10 \%$ in the rate of caries arrest (proportion of active caries) that has become hardened at a certain time point) between treatment groups was considered to be clinically significant. Based on a $5 \%$ statistical significant level and an $80 \%$ power, 360 decayed tooth surfaces would be required. A recent survey in Hong Kong found that on average a child with ECC has approximately four caries lesions. ${ }^{13}$ Thus, 90 children were needed in each group. Anticipating a $10 \%$ drop-out rate, a total of 304 children were recruited at baseline.

Collected data were entered into computer and analyzed using the software SPSS 20.0 for Windows (SPSS Inc., Chicago, USA) and WinBUGS 1.4. ${ }^{14}$ An intention-to-treat analysis was undertaken. Intra-examiner agreement in the diagnosis of dental caries was assessed by Cohen's Kappa statistics. Chi-square test and ANOVA were performed, when appropriate, to assess the differences between groups with regards to their baseline demographic background, oral health related behaviors and dmfs score. The level of statistical significance for all tests was set at 0.05 .

Since more than one caries lesion were chosen from one child, survival analysis using the Bayesian approach to analyze a set of multilevel clustered interval-censored data was used to assess the relative effectiveness of SDF solution and $\mathrm{NaF}$ varnish in arresting dentine caries. 
The analysis was carried out using the software WinBUGS, version 1.4. A two-level model was used, with tooth surface as level 1 and child as level 2. Time to arrest of caries was the outcome measure of effectiveness. The shorter the time, the more effective was the intervention to arrest active dentine caries. Since the children were examined every 6 months, the time intervals were grouped into 0-6, 6-12 and 12-18 months. In the estimation of parameters, the first 5,000 simulations were treated as burn-ins and discarded, while the estimation was based on the next 10,000 simulations. Besides treatment group assignment, other variables such as demographic characteristics (gender and age), baseline dmfs score, frequency of tooth brushing, use of fluoride toothpaste, snacking habits, location of the treated caries lesion (tooth type and tooth surface) and presence of plaque on lesion surface were also included in the model as covariates. The effects were considered statistically not significant if the $95 \%$ credible intervals for $\beta$ include 0 or, equivalently, the $95 \%$ credible interval for relative risk (RR) included $1 .{ }^{15}$

\section{Results}

A total of 304 children (183 boys and 121 girls) with 1670 tooth surfaces with active dentine caries received treatment at baseline. Their mean age was $41 \pm 4$ months. There were 100, 97 and 107 children in Groups 1, 2 and 3, respectively, and the respective numbers of treated active caries lesions in the three groups were 581, 488 and 601 (Figure 1). Most (>80\%) of the participants brushed their teeth with fluoridated toothpaste once a day or more often. Around half of the included teeth were upper anterior teeth and the most commonly involved surfaces were mesial surfaces (32\%) and occlusal surfaces (25\%). The mean dmft and dmfs scores at baseline were $4.4 \pm 3.4$ and $6.7 \pm 7.0$, respectively. No statistically significant differences were found among the three groups regarding the participants' background, oral health related behaviors, oral hygiene status and baseline caries status (Table 1).

After 18 months, 275 (91\%) children remained in the study, 89, 89 and 97 children in Groups 1, 2 and 3, respectively. The drop-out rates among the three groups were similar $\left(\chi^{2}\right.$ test, $\mathrm{p}>0.05$ ). The differences in the parameters between the initial group of 304 children and the 275 children who remained in the study were not statistically significant ( $p>0.05)$. Intraobserver reliability was good with Kappa statistic values greater than 0.9 in the baseline and follow-up examinations. 
As shown in Table 2, the 18-month caries arrest rates of the treated lesions were 40\%, $35 \%$ and $27 \%$ in Group 1 (SDF once a year), Group 2 (SDF intensive) and Group 3 (NaF intensive), respectively ( $\chi^{2}$ test, $\left.\mathrm{p}<0.001\right)$. In all of the follow-up examinations, lesions treated with the intensive $\mathrm{NaF}$ varnish applications, i.e. 3 weekly applications at baseline, (Group 3) had lower caries arrest rates than those treated with SDF solution regardless the application protocol. At the 6-month and the 12-month follow-up, the lesions that had received 3 weekly applications of SDF solution at baseline (Group 2) had higher caries arrest rates than those that had received only one SDF application at baseline (Group 1).

Results of the multi-level survival analysis showed that the clustering effect among the arrest times of the caries lesions from the same child was strong $\left(\sigma^{2}\right.$ child $=1.68$; $95 \%$ credible interval $=1.103-2.568$ ), and the estimated intra-cluster correlation coefficient was 0.505 (Table 3). Four variables, namely treatment group, presence of plaque on lesion, tooth type and tooth surface affected the time to arrest of active caries, while the other variables including demographic background, oral health related behaviors, and baseline caries experience were not statistically significant. The two SDF solution application protocols (Group 1 and Group 2) significantly shortened the time to caries arrest when compared to the $\mathrm{NaF}$ varnish application protocol (Group 3). When the two SDF solution application protocols were compared, no significant differences at the 18-month follow-up were found. Effects of tooth type, tooth surface and presence of plaque on lesion surface were statistically significant (Table 3). Lesions in anterior teeth had a higher chance to become arrested at 18 months compared to those in posterior teeth. Regarding the type of tooth surface, lesions in buccal/lingual surfaces had a higher chance to become arrested than those in occlusal surfaces. Absence of plaque on the lesion surface significantly shortened the time to caries arrest. Except the blackening of carious lesions, especially those treated with SDF, no adverse effects on the treated teeth and soft tissues were found.

\section{Discussion}

Since the children participating in this study were at high risk of dental caries and had active dentine caries, fluoride treatments were provided to all participants. Thus, the current study was unable to compare the effects of the three intervention methods with a negative control group. Based on the study findings and with limitations, the present study supports the use of SDF solution (either 3 weekly applications at baseline or every 12 months) to arrest 
dentine caries in preschool children due to its higher effectiveness, compared to intensive $\mathrm{NaF}$ varnish application (3 weekly applications at baseline). These findings are consistent with earlier clinical observations ${ }^{5}$ and findings of recent review ${ }^{16}$ which support the effectiveness of SDF in arresting dentine caries in children. Laboratory studies found that SDF could inhibit demineralization, preserve collagen from degradation in demineralized dentine and increase the hardness of treated dentine caries. ${ }^{17,18}$ Regarding the effectiveness of different of SDF application protocols, this study showed that a one-off intensive SDF application protocol with 3 weekly applications can lead a higher proportion of active dentine lesions in primary teeth being arrested in the short-term (within a year) and provide an outcome not significantly different from that of annually repeated applications in the longer term (18 months). It seems that either of the two SDF application protocols can be adopted when a non-restorative treatment approach is chosen for managing dentine caries in primary teeth. In choosing an appropriate SDF application protocol the special needs of the particular population and the resources available, such as dental care providers, should be considered. For children in mobile populations, a one-off intensive SDF application protocol is probably a more practical alternative to the other dental caries control protocols that require regular dental attendance for receiving intervention. It should be noted that this study was conducted among high caries risk preschool children living in a fluoridated area. The study results need to be interpreted with caution as the findings may not be transferable to other population groups, such as children living in non-fluoridated areas.

Besides the study interventions, in this study effects of other possible confounding factors were also evaluated. Obviously, data from lesions in the same child's teeth were not independent and it was found that the correlation of the times to caries arrest obtained from the same child was quite strong. Data analysis that ignores the clustering effect would yield biased results and true differences would be obscured. In the present study, results of multi-level survival analysis confirmed the significant effect of tooth type, tooth surface and the presence of plaque on lesion surface on caries arrest rates. These findings are consistent with those from earlier studies ${ }^{6}$ which found that lesions in the anterior teeth or buccal/lingual surfaces had a higher chance to become arrested. Plaque accumulation on the lesion was found to be an important factor affecting the progression of carious lesions. Our findings support the concept that alteration of the biofilm will alter lesion formation and progression. ${ }^{19}$ Taken together, these results imply that plaque control at the lesion site is a crucial factor affecting the success of caries arrest treatment for active caries lesions in primary teeth. 
It has been found that the effect of professionally applied fluoride may decrease over time after application. ${ }^{7}$ On the contrary, in this study, the caries arrest rates among the lesions that had received three weekly applications of SDF solution (Group 2) or NaF varnish (Group 3) at baseline gradually increased over time after treatment even without annual re-application of the study fluoride agents. The positive results over time may be due to the effect of other sources of fluoride and the children's development. As stated previously, the drinking water in Hong Kong is fluoridated and the majority of children in this study used fluoridated toothpaste during their daily tooth brushing. As the study children became older, from 3 to 5 years old, it is likely that they had improved their manual dexterity and were able to carry out more effective cleaning of their teeth. They may also have better compliance with advices on regular tooth brushing, resulting in better plaque control.

In this study, the caries arrest rates of SDF groups were found to be somewhat lower than those of other studies. ${ }^{7,20}$ There are several possible explanations for this observation. In the study kindergartens, the dentists who applied the fluoride agents in this study also had to provide basic dental care to the other children who were not involved in this study. As a result, they could not afford to spend much time on the topical fluoride application procedures and thus the application time of fluoride agent in this study (around 10 seconds per involved tooth) may be shorter than that in other studies. This probably had affected the efficacy of the fluoride agents in arresting dentine caries. Besides, the assessment criteria used in the present study were very strict. Caries arrest was not recorded if the lesion was not totally arrested, i.e. if any area in the lesion was not hard to gentle probing. Another possible explanation may be related to the stringency of the examiner. Probably, there are differences between examiners in classifying caries status because of the different clinical experience and opinions of the examiners. Judgement on the dullness of lesion appearance and the tactile sensation with probing involve a relatively high degree of subjectivity. ${ }^{21}$ However, up to date, visual-tactile inspection is the only validated method for assessing caries lesion in a single session. ${ }^{22}$ Further research to develop an accurate, objective and reproducible lesion activity measurement in a clinical setting is needed.

Black stain on the dentine surface of the arrested caries lesions, which is a known side effect of SDF, was found in the SDF groups in the present study. The advantages and disadvantages of using SDF have been elucidated in previous research. ${ }^{5,7,16}$ The acceptance of 
having black stains on some parts of the teeth varies between people and this may be regarded as undesirable for the more aesthetic-oriented patients and dental professionals. Studies on how to avoid or reduce the amount of black staining of caries lesions treated by SDF are needed.

The possibility of causing chronic and acute toxic side-effects through the use of SDF has been debated. ${ }^{23,24}$ In the present study, besides the black staining, no adverse effects were observed during the 18-month follow-up. With regard to the metabolism of fluoride, plasma fluoride levels increase rapidly after ingestion, reaching the peak within 20-60 minutes ${ }^{25}$ and drop to baseline plasma levels within 3 to 11 hours, depending on the amount ingested ${ }^{26}$. Due to the rapid absorption and excretion of fluoride, the risk of fluoride overdose by multiple applications with weekly interval is small. Regarding the potential risk associated with silver ingestion, preliminary data in adults showed that occasional use of SDF was well below the concentration associated with toxicity. ${ }^{27}$ However, without data on young children, this possibility cannot be excluded and one needs to pay attention to the safety aspect when applying high concentration fluoride and silver agents to young children.

\section{Conclusion}

Among high caries risk preschool children living in a fluoridated area, annual or three consecutive weekly applications of SDF solution is more effective in arresting dentine caries in primary teeth than three consecutive weekly applications of $\mathrm{NaF}$ varnish.

\section{Acknowledgement}

The authors thank Dr. May Wong and Ms. Samantha Li for their statistical advice. This study was funded by the University of Hong Kong and there is no conflict of interest. 


\section{References}

1. Edelstein BL. Solving the problem of early childhood caries: a challenge for us all. Archives of Pediatrics \& Adolescent Medicine 2009;163:667-8.

2. Finucane D. Rationale for restoration of carious primary teeth: A review. European Archives of Paediatric Dentistry 2012 Dec;13:281-92.

3. Chu CH, Ho PL, Lo EC. Oral health status and behaviours of preschool children in Hong Kong. BMC Public Health 2012;12:767.

4. Frencken JE, Peters MC, Manton DJ, Leal SC, Gordan VV, Eden E. Minimal intervention dentistry for managing dental caries - a review: report of a FDI task group. International Dental Journal 2012;62:223-43.

5. Chu CH, Lo EC, Lin HC. Effectiveness of silver diamine fluoride and sodium fluoride varnish in arresting dentin caries in Chinese pre-school children. Journal of Dental Research 2002;81:767-70.

6. Zhi QH, Lo EC, Lin HC. Randomized clinical trial on effectiveness of silver diamine fluoride and glass ionomer in arresting dentine caries in preschool children. Journal of Dentistry 2012;40:962-7.

7. Yee R, Holmgren C, Mulder J, Lama D, Walker D, van Palenstein Helderman W. Efficacy of silver diamine fluoride for Arresting Caries Treatment. Journal of Dental Research 2009;88:644-7.

8. Llodra JC, Rodriguez A, Ferrer B, Menardia V, Ramos T, Morato M. Efficacy of silver diamine fluoride for caries reduction in primary teeth and first permanent molars of schoolchildren: 36-month clinical trial. Journal of Dental Research 2005;84:721-4.

9. Braga MM, Mendes FM, De Benedetto MS, Imparato JC. Effect of silver diammine fluoride on incipient caries lesions in erupting permanent first molars: a pilot study. Journal of Dentistry for Children 2009;76:28-33.

10. Weinstein P, Spiekerman C, Milgrom P. Randomized equivalence trial of intensive and semiannual applications of fluoride varnish in the primary dentition. Caries Research 2009;43:484-90.

11. International Caries detection and Assessment System Coordinating Committee. Criteria Manual International Caries Detection and Assessment System (ICDASII). https://www.icdas.org/uploads/ICDAS\%20Criteria\%20Manual\%20Revised\%202009 _2.pdf. [Accessed 4 July 2013] 
12. Yee R, Holmgren C, Mulder J, Lama D, Walker D, Helderman WVP. Efficacy of silver diamine fluoride for arresting caries treatment. Journal of Dental Research 2009;88:644-7.

13. Department of Health, Government of the Hong Kong Special Administrative Region. Oral Health Survey 2011. Hong Kong; 2013.

14. Spiegelhalter D, Thomas A, Best N, Lunn D. WinBUGS Version 1.4 user manual.Cambridge: MRC Biostatistics Unit; 2003.

15. Wong MCM, Lam KF, Lo ECM. Bayesian analysis of clustered interval-censored data. Journal of Dental Research 2005;84:817-21.

16. Rosenblatt A, Stamford TC, Niederman R. Silver diamine fluoride: a caries "silverfluoride bullet". Journal of Dental Research 2009;88:116-25.

17. Mei ML, Ito L, Cao Y, Li QL, Lo EC, Chu CH. Inhibitory effect of silver diamine fluoride on dentine demineralisation and collagen degradation. Journal of Dentistry 2013;41:809-17.

18. Chu $\mathrm{CH}$, Lo ECM. Microhardness of dentine in primary teeth after topical fluoride applications. Journal of Dentistry 2008;36:387-91.

19. Kidd E. The implications of the new paradigm of dental caries. Journal of Dentistry 2011;39 (Suppl 2):S3-8.

20. dos Santos VE, Jr., Vasconcelos Filho A, Targino AG, Flores MA, Galembeck A, Caldas AF, Jr., et al. A new "silver-bullet" to treat caries in children--nano silver fluoride: a randomised clinical trial. Journal of Dentistry 2014;42:945-51.

21. Ekstrand KR, Zero DT, Martignon S, Pitts NB. Lesion activity assessment. Monographs in Oral Science 2009;21:63-90.

22. Braga MM, Ekstrand KR, Martignon S, Imparato JC, Ricketts DN, Mendes FM. Clinical performance of two visual scoring systems in detecting and assessing activity status of occlusal caries in primary teeth. Caries Research 2010;44:300-8.

23. Neesham DC. Fluoride concentration in AgF and dental fluorosis. Australian Dental Journal 1997;42:268-9.

24. Gotjamanos T. Safety issues related to the use of silver fluoride in paediatric dentistry. Australian Dental Journal 1997;42:166-8.

25. Buzalaf MA, Whitford GM. Fluoride metabolism. Monographs in Oral Science 2011;22:20-36. 26.

26. Whitford GM. The metabolism and toxicity of fluoride. Monographs in Oral Science 1996;16:1-153. 
27. Vasquez E, Zegarra G, Chirinos E, Castillo JL, Taves DR, Watson GE, et al. Short term serum pharmacokinetics of diammine silver fluoride after oral application. BMC Oral Health 2012;12:60. 


\section{Figure legend}

Figure 1. Flow of subjects over the18-month study period

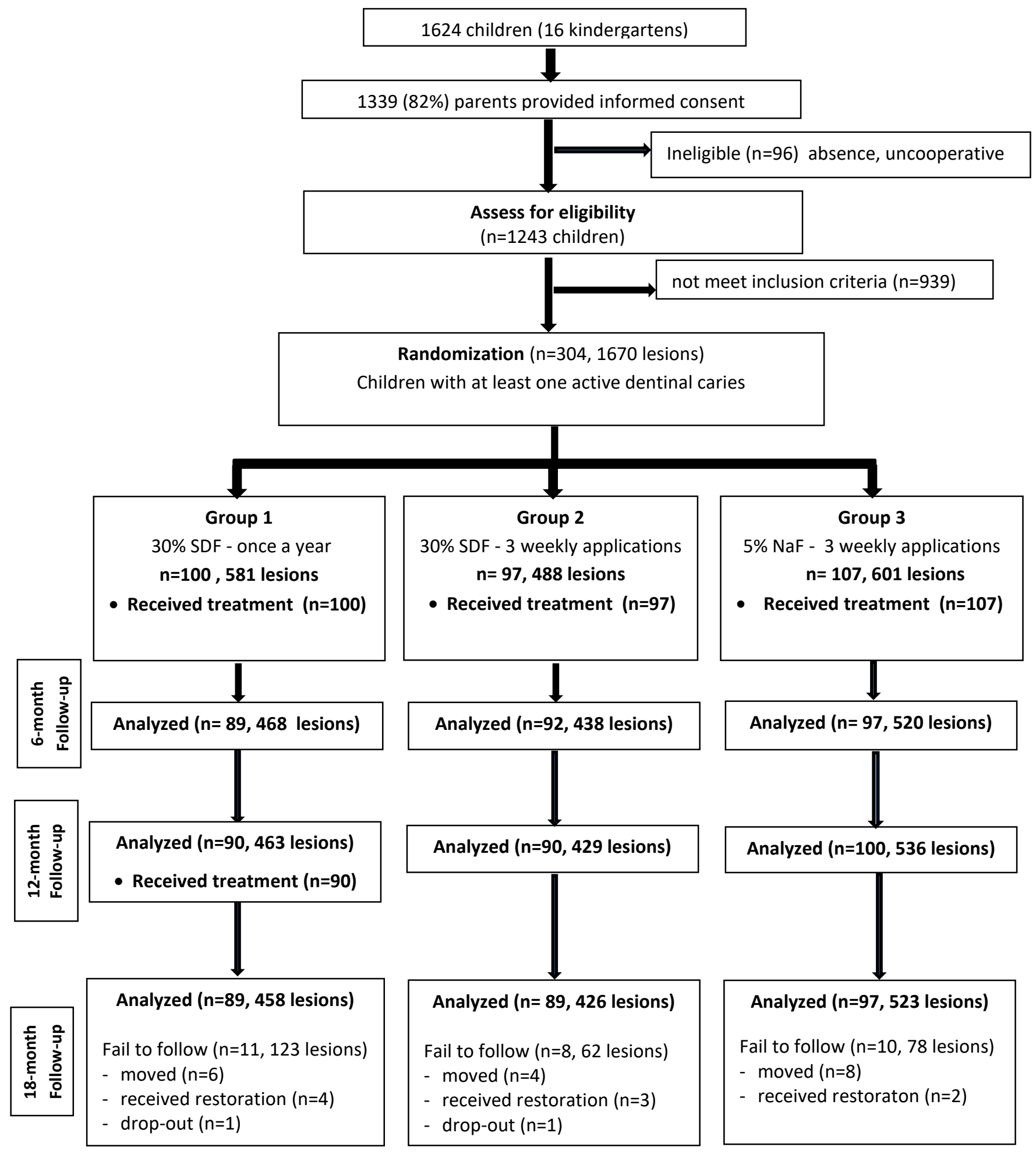

Figure 1. Flow of subjects over the18-month study period 
Table 1 Background information of children in the three study groups at baseline.

\begin{tabular}{|c|c|c|c|}
\hline & $\begin{array}{l}\text { Group1 SDF } \\
\text { (once a year) }\end{array}$ & $\begin{array}{c}\text { Group 2 SDF } \\
\text { (3 applications } \\
\text { weekly interval) }\end{array}$ & $\begin{array}{l}\text { Group } 3 \text { NaF } \\
\text { (3 applications } \\
\text { weekly interval) }\end{array}$ \\
\hline No. of children (lesions) & $\mathrm{n}=100(581)$ & $\mathrm{n}=97(488)$ & $\mathrm{n}=107(601)$ \\
\hline Mean age (month) & 41 & 40 & 41 \\
\hline $\begin{array}{l}\text { Gender } \\
\text { Male } \\
\text { Female }\end{array}$ & $\begin{array}{l}60 \% \\
40 \%\end{array}$ & $\begin{array}{l}58 \% \\
42 \%\end{array}$ & $\begin{array}{l}63 \% \\
37 \%\end{array}$ \\
\hline $\begin{array}{l}\text { Toothbrushing } \\
\text { Less than once a day } \\
\text { Once a day } \\
\text { Twice or more a day }\end{array}$ & $\begin{array}{l}14 \% \\
48 \% \\
38 \%\end{array}$ & $\begin{array}{l}16 \% \\
38 \% \\
47 \%\end{array}$ & $\begin{array}{l}18 \% \\
39 \% \\
43 \%\end{array}$ \\
\hline $\begin{array}{l}\text { Fluoride toothpaste } \\
\text { Yes } \\
\text { No }\end{array}$ & $\begin{array}{l}87 \% \\
13 \%\end{array}$ & $\begin{array}{l}80 \% \\
20 \%\end{array}$ & $\begin{array}{l}83 \% \\
17 \%\end{array}$ \\
\hline $\begin{array}{l}\text { Family monthly income } \\
\text { Low }(<\mathrm{HK} \$ 15 \mathrm{k}) \\
\text { Middle }(\mathrm{HK} \$ 15-25 \mathrm{k}) \\
\text { High (>HK\$ 25k) }\end{array}$ & $\begin{array}{l}62 \% \\
25 \% \\
13 \%\end{array}$ & $\begin{array}{l}58 \% \\
19 \% \\
23 \%\end{array}$ & $\begin{array}{l}55 \% \\
26 \% \\
19 \%\end{array}$ \\
\hline Visible Plaque Index & $58 \%$ & $56 \%$ & $55 \%$ \\
\hline $\begin{array}{l}\text { Dental caries status } \\
\text { Mean dmft (SD) } \\
\text { Mean dmfs (SD) }\end{array}$ & $\begin{array}{l}4.5(3.4) \\
6.8(6.6)\end{array}$ & $\begin{array}{l}4.2(3.2) \\
6.1(6.6)\end{array}$ & $\begin{array}{l}4.6(3.7) \\
7.1(7.9)\end{array}$ \\
\hline $\begin{array}{l}\text { Tooth type included } \\
\text { Anterior teeth } \\
\text { Posterior teeth }\end{array}$ & $\begin{array}{l}64 \% \\
36 \%\end{array}$ & $\begin{array}{l}66 \% \\
34 \%\end{array}$ & $\begin{array}{l}64 \% \\
36 \%\end{array}$ \\
\hline $\begin{array}{l}\text { Tooth surfaces included } \\
\text { Occlusal } \\
\text { Proximal } \\
\text { Buccal/Lingual }\end{array}$ & $\begin{array}{l}25 \% \\
45 \% \\
30 \%\end{array}$ & $\begin{array}{l}25 \% \\
46 \% \\
29 \%\end{array}$ & $\begin{array}{l}26 \% \\
49 \% \\
25 \%\end{array}$ \\
\hline
\end{tabular}


Table 2 Caries arrest rates of cavitated dentine lesions (ICDAS 5 or 6) at 6-, 12-, 18-month follow-up in the three treatment groups (at surface level)

\begin{tabular}{|c|c|c|c|}
\hline & $\begin{array}{l}\text { 6-month } \\
(n=1426)\end{array}$ & $\begin{array}{l}\text { 12-month } \\
(\mathrm{n}=1428)\end{array}$ & $\begin{array}{l}\text { 18-month } \\
(\mathrm{n}=1407)\end{array}$ \\
\hline $\begin{array}{l}\text { Group1 SDF } \\
\text { (once a year) }\end{array}$ & $\begin{array}{c}18 \% \\
(82 / 468)\end{array}$ & $\begin{array}{c}20 \% \\
(91 / 463)\end{array}$ & $\begin{array}{c}40 \% \\
(181 / 458)\end{array}$ \\
\hline $\begin{array}{l}\text { Group } 2 \text { SDF } \\
\text { (3x with weekly interval) }\end{array}$ & $\begin{array}{c}31 \% \\
(134 / 438)\end{array}$ & $\begin{array}{c}28 \% \\
(118 / 429)\end{array}$ & $\begin{array}{c}35 \% \\
(149 / 426)\end{array}$ \\
\hline \multirow[t]{2}{*}{$\begin{array}{l}\text { Group } 3 \mathrm{NaF} \\
\text { (3x with weekly interval) }\end{array}$} & $\begin{array}{c}10 \% \\
(54 / 520)\end{array}$ & $\begin{array}{c}13 \% \\
(71 / 536)\end{array}$ & $\begin{array}{c}27 \% \\
(141 / 523)\end{array}$ \\
\hline & $P<0.001$ & $P<0.001$ & $\mathrm{P}<0.001$ \\
\hline
\end{tabular}


Table 3 Multi-level survival analysis with WinBUGS- Results from 10,000 stimulations after 5000 burn-ins

\begin{tabular}{|c|c|c|c|c|}
\hline & \multicolumn{2}{|c|}{ Log hazard ratio } & \multicolumn{2}{|c|}{ Hazard ratio } \\
\hline & Median* & 95\% C.I. & Median* & 95\% C.I. \\
\hline \multicolumn{5}{|l|}{ Treatment } \\
\hline Gp.1 vs Gp. 3 & 1.15 & $(0.50,1.74)$ & 3.16 & $(1.66,5.72)$ \\
\hline Gp. 2 vs Gp. 3 & 1.64 & $(1.00,2.28)$ & 5.16 & $(2.72,9.81)$ \\
\hline Gp. 1 vs Gp. 2 & -0.49 & $(-1.10,0.06)$ & 0.61 & $(0.33,1.07)$ \\
\hline \multicolumn{5}{|l|}{ Tooth type } \\
\hline Anterior vs Posterior & 1.46 & $(0.97,2.01)$ & 4.21 & $(2.64,7.49)$ \\
\hline \multicolumn{5}{|l|}{ Surface type } \\
\hline Proximal vs Occlusal & -0.37 & $(-0.98,0.20)$ & 0.69 & $(0.37,1.22)$ \\
\hline Buccal/Lingual vs Occlusal & 0.96 & $(0.36,1.55)$ & 2.59 & $(1.43,4.72)$ \\
\hline Absence of Plaque & 4.46 & $(4.01,4.97)$ & 86.83 & $(55.37,144.2)$ \\
\hline$\sigma^{2}$ child & 1.680 & $(1.103,2.568)$ & & \\
\hline Corr subject & 0.505 & $(0.401,0.610)$ & & \\
\hline
\end{tabular}

Median = median of the estimates drawn from the posterior distributions

Hazard ratio $>1$ corresponds to a higher chance of arrest of active dentine caries and then a shorter arrest time

95\% C.I. $=95 \%$ credible interval 